\title{
To Excavate Biomarkers Predictive of the Response for Capecitabine plus RAD001 through Nanostring-Based Multigene Assay in Advanced Gastric Cancer Patients
}

\author{
Hansang Lee', Jeeyun Lee ${ }^{1,2}$, Insuk Sohn³ ${ }^{3}$, Se Hoon Park ${ }^{1,2}$, Joon Oh Park ${ }^{1,2}$, Young Suk Park ${ }^{1,2}$, \\ Kyoung-Mee Kim ${ }^{4}$, Won Ki Kang1,2, Seung Tae Kim ${ }^{1,2}{ }^{\square}$ \\ 1. Division of Hematology-Oncology, Department of Medicine, Samsung Medical Center, Sungkyunkwan University School of Medicine, Seoul, Korea; \\ 2. Gastric Cancer Center, Samsung Medical Center, Sungkyunkwan University School of Medicine, Seoul, Korea; \\ 3. Samsung Cancer Research Institute, Seoul, Korea; \\ 4. Department of Pathology, Samsung Medical Center, Sungkyunkwan University School of Medicine, Seoul, Korea.
}

$\square$ Corresponding author: Seung Tae Kim, M.D., Ph.D., Division of Hematology/Oncology, Department of Medicine, Samsung Medical Center, Sungkyunkwan University School of Medicine, 81 Irwon-ro, Gangnam-gu, Seoul 135-710, Korea. Tel: +82-2-3410-0297; Fax: +82-2-3410-1754; Email: shty1@skku.edu.

(c) Ivyspring International Publisher. Reproduction is permitted for personal, noncommercial use, provided that the article is in whole, unmodified, and properly cited. See http://ivyspring.com/terms for terms and conditions.

Received: 2016.06.20; Accepted: 2016.09.18; Published: 2016.10.25

\begin{abstract}
Comprehensive characterization of individual patients' tumour is important to realize personalized medicine. Here, we investigate to identify subsets that benefit from capecitabine plus RAD001 in advanced gastric cancer (GC) patients by comprehensive high-throughput genomic analysis (nCounter assay). Archival tumour tissue blocks, if possible, were collected at phase II trial of capecitabine plus RAD001 in 47 refractory GC patients (at clinicaltrials.gov NCT\#01099527). A total of 42 formalin-fixed, paraffin-embedded (FFPE) tumour samples were available for nanostring based-multigene Assay. An nCounter assay of 519 kinase panels has been used. We performed correlation analyses between expression levels of kinase genes and response for capecitabine plus RAD001. Among 42 patients with An nCounter assay of 519 kinase panels, 4 patients achieved confirmed partial response and 15 patients revealed stable disease, resulting in an overall response rate (ORR) of $9.5 \%$. No difference in ORR was observed in terms of gender, performance status, primary tumour site, gastric resection, histologic subtype, Lauren classification, No. of metastatic site and No. of chemotherapy. In subgroups with response for capecitabine plus RAD001, there is significant overexpression of 6 genes among 519 kinase gene such as EPHA2 $(P=0.0025), P I M 1(P=0.0031), K S R 1$ $(P=0.0033)$, and EIF2AK4 $(P=0.0046)$ that are related to the activation of $m$ TOR signalling. This study is first report that investigated to identify biomarkers predictive of the response for RAD001 containing treatment in refractory GC patients, by comprehensive high-throughput genomic analysis (nCounter assay).
\end{abstract}

Key words: Nanostring, RAD001, gastric cancer

\section{Introduction}

Gastric cancer (GC) is still the fourth most common cancer and the second most common cause of annual cancer related deaths worldwide [1, 2]. For patients with recurrent or metastatic gastric cancer, chemotherapy is the standard care, but the response rate is still below $50 \%$ and the duration of response is as short as a few months [3]. Salvage chemotherapy in patients who have failed one cytotoxic chemotherapy was recently proven to be efficacious in gastric cancer by our group. Nevertheless, the median overall survival for docetaxel was 5.2 months and irinotecan was 6.5 months [4]. Hence, salvage treatment needs to be aggressively investigated in GC patients, especially those who have failed first-line chemotherapy.

Phphatidylinositol 3 kinase (PI3K)/Akt and mammalian target of rapamycin (mTOR) are activated in $30 \%$ and $60 \%$ of human gastric carcinoma, respectively $[5,6]$. mTOR, a serine/threonine kinase, 
is thought to play a central role in regulating cell growth, proliferation, cellular metabolism and angiogenesis [7]. Inhibition of the mTOR signaling as a new therapeutic target is an active area of research. Currently, everolimus (RAD001) is the only oral mTOR imhibitor that has been investigated in phase I/II / III clinical trials of patients with advanced GC [8-11]. Furthermore, based on preclinical studies showing synergistic effects with some cytotoxic agents including 5-fluorouracil (5-FU), some clinical studies have been evaluating RAD001 in patients with GC in combination with chemotherapeutic agents [12-14]. Our group also conducted phase II trial of capecitabine plus RAD001 in refractory GC patients [12]. Irrespective of whether these trials used RAD001 met their primary end points, these commonly showed that treatment including RAD001 had the effectiveness in small subset of GC patients who were pretreated. Thus, identification of specific biomarkers for heterogeneous patient subpopulation with advanced GC may help define those patients who would receive the most benefit from RAD001 treatment. However, despite many efforts, biomarkers predictive of therapeutic success for RAD001 have not been determined in GC patients.

Comprehensive characterization of individual patient's tumor is needed for realization of personalized strategies for each patient. We chose to use nCounter platform (Nanostring Technologies, Seattle, WA) due to its ability to interrogate expression levels of up to 800 genes using total RNA extracted from FFPET in a single tube reaction [15]. One of the pre-built multigene kinase assay surveys known cancer kinase genes. Recently, we confirmed that there was high concordance in assessment of triple markers (EGFR, HER2, and MET) in AGC between IHC test and nCounter assay with high sensitivity and high specificity. We investigated to identify subsets that benefit from capecitabine plus RAD001 in advanced gastric cancer (GC) patients with nCounter assay as reliable high-throughput genomic analyser.

\section{Methods}

\section{Patients and samples}

Between March 2010 and June 2012, a phase II trial for capecitabine plus RAD001 in AGC patients had been conducted in our institute, Samsung Medical Center, Seoul, Korea (clinicaltrials.gov NCT\#01099527) [12]. GC tissues were collected from 42 of all 47 patients enrolled in the above clinical trial. The process for acquisition of tissue was approved by the institutional review board of the Samsung Medical Center. All study participants provided written informed consent form recommended by the IRB. For all cases, we reviewed the data for age at diagnosis, gender, ECOG (Eastern Cooperative Oncology Group) performance status, primary site of GC, prior gastric resection, histologic subtype, Lauren classification, numbers of involved organ, metastatic site, and prior chemotherapy. All H\&E stained slides were reviewed and representative areas were carefully selected and marked on all paraffin blocks.

\section{Nanostring-based multigene assay}

Nanostring-Based Multigene Assay was applied to 42 of 47 patients enrolled in a phase II trial for capecitabine plus RAD001. Total RNA was extracted from 1-2 sections of 4- $\mu \mathrm{m}$ thick formalin-fixed, paraffin-embedded (FFPE) tumour sections from representative tumor blocks using the High Pure RNA Paraffin kit (Roche Diagnostic, Mannheim, Germany) after removing non-tumor elements by manual macrodissection guided by hematoxylin and eosin stained slides. For Nanostring-Based Multigene Assay (nCounter assay), $100 \mathrm{ng}$ of total RNA was hybridized with the custom designed code set of 519 genes for 18 hours at $65^{\circ} \mathrm{C}$ and processed according to manufacturer's instruction [15].

\section{Statistical analysis}

Standard descriptive and analytical methods were used to describe the patients' characteristics, and clinical outcomes. The tumor response was classified on the basis of Response Evaluation Criteria in Solid Tumors (RECIST) criteria, version 1.0 guidelines. The association of treatment response with various clinic-pathologic features was analyzed using the chi-square test and Fisher's exact test. In order to identify differentially expressed genes related to the response for capecitabine plus RAD001, we performed t-tests with 10,000 per mutations.

\section{Results}

\section{Clinicopathological features}

In this study, we analysed 42 with tumour sample available for nanostring based-multigene Assay among 47 AGC patients enrolled in a phase II trial for capecitabine plus RAD001 as salvage therapy between March 2010 and June 2012 (Figure 1). As are summarized in Table 1, patients composed of 31 $(73.8 \%)$ male and $11(26.2 \%)$ female with median age 52 years (range 37-78). Most of the patients (97.6\%) had a good performance status (ECOG 0 or 1 ) and over half of patients $(64.3 \%)$ had gastric resection. Most patients (81\%) had 2 or more metastatic lesion and $90.5 \%$ received 3 or more palliative chemotherapy including capecitabine pus RAD001. 
Table 1. Clinicopathological characteristics of patients with advanced gastric cancer (GC) in this study.

\begin{tabular}{ll}
\hline Characteristics & No. of patients (\%) (N=42) \\
\hline Age (years), Median (range) & $52.0(37.0-78.0)$ \\
Sex & $31(73.8)$ \\
Male & $11(26.2)$ \\
Female & \\
ECOG PS & $3(7.1)$ \\
0 & $38(90.5)$ \\
1 & $1(2.4)$ \\
2 & \\
Primary site of GC & $16(38.1)$ \\
Body/Fundus & $21(50.0)$ \\
Antrum & $2(4.8)$ \\
Multifocal & \\
Gastric resection & $27(64.3)$ \\
Positive & $15(35.7)$ \\
Negative & \\
Histology & $33(78.6)$ \\
Tubular adenocarcinoma & $7(16.7)$ \\
Signet ring cell carcinoma & $2(4.8)$ \\
Others & \\
Lauren classification & $15(35.7)$ \\
Intestinal & $11(26.2)$ \\
Diffuse & \\
No. of involved organs & $8(19.0)$ \\
1 & $13(31.0)$ \\
2 & $15(35.7)$ \\
3 & $6(14.3)$ \\
$\geq 4$ & $19(45.2)$ \\
Metastatic sites & $20(47.6)$ \\
Liver & $17(40.5)$ \\
Abdominal LN & $5(11.9)$ \\
Peritoneal seeding & $1(2.4)$ \\
Lung & $1(2.4)$ \\
Cervical LN & $4(9.5)$ \\
Bone & $11(26.2)$ \\
No. of palliative chemotherapy & $6(14.3)$ \\
$1-2$ & \\
3 & \\
4 & \\
$\geq 5$ & \\
\hline
\end{tabular}

\section{Treatment efficacy}

Among 42 patients available for an nCounter assay, 4 patients achieved confirmed partial response and 15 patients revealed stable disease, resulting in an overall response rate (ORR) of 9.5\% (Table 2). Disease control rate was $45.2 \%$. Three patients were not available for response evaluation. No difference in ORR was observed in terms of gender, performance status, primary tumour site, gastric resection, histologic subtype, Lauren classification, No. of metastatic site and No. of chemotherapy (Table 3).

\section{Nanostring-based multigene assay to select responder to capecitabine plus RAD001}

We evaluated the expression-nature of kinase genes by Nanostring-Based Multigene Assay in 42 patients. Among expression nature of 519 kinase genes, overexpressed kinase genes related to the response for capecitabine plus RAD001 were sorted while changing the weight of P-value such as 0.05 , 0.01 , and 0,005 . When the cut-off of P-value for the significance is 0.005 (Table 4), there is significant overexpression of 4 genes among 519 kinase gene such as EPHA2 ( $\mathrm{P}=0.0025), \mathrm{PIM} 1(\mathrm{P}=0.0031)$, KSR1 $(\mathrm{P}=0.0033)$, and EIF2AK4 $(\mathrm{P}=0.0046)$ that are related to the activation of mTOR-signaling (Figure 2).

Table 2. Best overall response.

\begin{tabular}{lll}
\hline Best Overall Response & No. of patients & $\%$ \\
\hline CR & - & - \\
PR & 4 & $9.5 \%$ \\
SD & 15 & $35.7 \%$ \\
PD & 20 & $47.6 \%$ \\
Not evaluable & 3 & $7.1 \%$ \\
\hline
\end{tabular}

Table 3. The efficacy for capecitabine plus RAD001 according to clinicopathological characteristics of patients.

\begin{tabular}{|c|c|c|c|}
\hline Characteristics & $\begin{array}{l}\text { No. of patients } \\
(\mathrm{N}=42)\end{array}$ & $\begin{array}{l}\text { Responder for } \\
\text { Capecitabine/RAD001 }\end{array}$ & P-value \\
\hline Sex & & & 0.558 \\
\hline Male & 31 & 4 & \\
\hline Female & 11 & 0 & \\
\hline ECOG PS & & & 1.000 \\
\hline 0 & 3 & 0 & \\
\hline 1 & 38 & 4 & \\
\hline 2 & 1 & 0 & \\
\hline Primary site of GC & & & 0.435 \\
\hline Body/Fundus & 16 & 3 & \\
\hline Antrum & 21 & 1 & \\
\hline Multifocal & 2 & 0 & \\
\hline Gastric resection & & & 1.000 \\
\hline Positive & 27 & 3 & \\
\hline Negative & 15 & 1 & \\
\hline Histology & & & 0.097 \\
\hline Tubular adenocarcinoma & 33 & 2 & \\
\hline Signet ring cell carcinoma & 7 & 1 & \\
\hline Others & 2 & 1 & \\
\hline \multicolumn{4}{|l|}{ Lauren classification } \\
\hline Intestinal & 15 & & \\
\hline Diffuse & 11 & & \\
\hline No. of involved organs & & & 0.176 \\
\hline 1 & 8 & 1 & \\
\hline 2 & 13 & 3 & \\
\hline 3 & 15 & 0 & \\
\hline$\geq 4$ & 6 & 0 & \\
\hline $\begin{array}{l}\text { No. of palliative } \\
\text { chemotherapy }\end{array}$ & & & 0.775 \\
\hline $1-2$ & 4 & 0 & \\
\hline 3 & 11 & 2 & \\
\hline 4 & 21 & 2 & \\
\hline$\geq 5$ & 6 & 0 & \\
\hline
\end{tabular}

Table 4. Profiles for kinase gene overexpression associated with the effect of capecitabine plus RAD001.

\begin{tabular}{|c|c|c|c|}
\hline & & Kinase gene expression & P-value \\
\hline \multirow{4}{*}{$\begin{array}{l}\text { Capecitabine plus } \\
\text { RAD001 }\end{array}$} & Response & EPHA2 & 0.0025 \\
\hline & & PIM1 & 0.0031 \\
\hline & & KSR1 & 0.0033 \\
\hline & & EIF2AK4 & 0.0047 \\
\hline
\end{tabular}




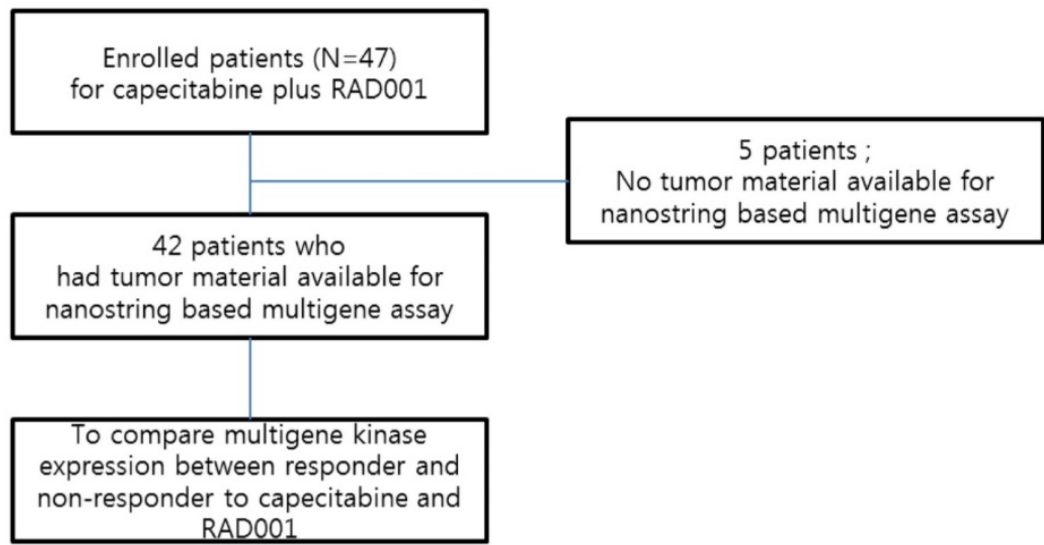

Figure 1. Consort diagram.

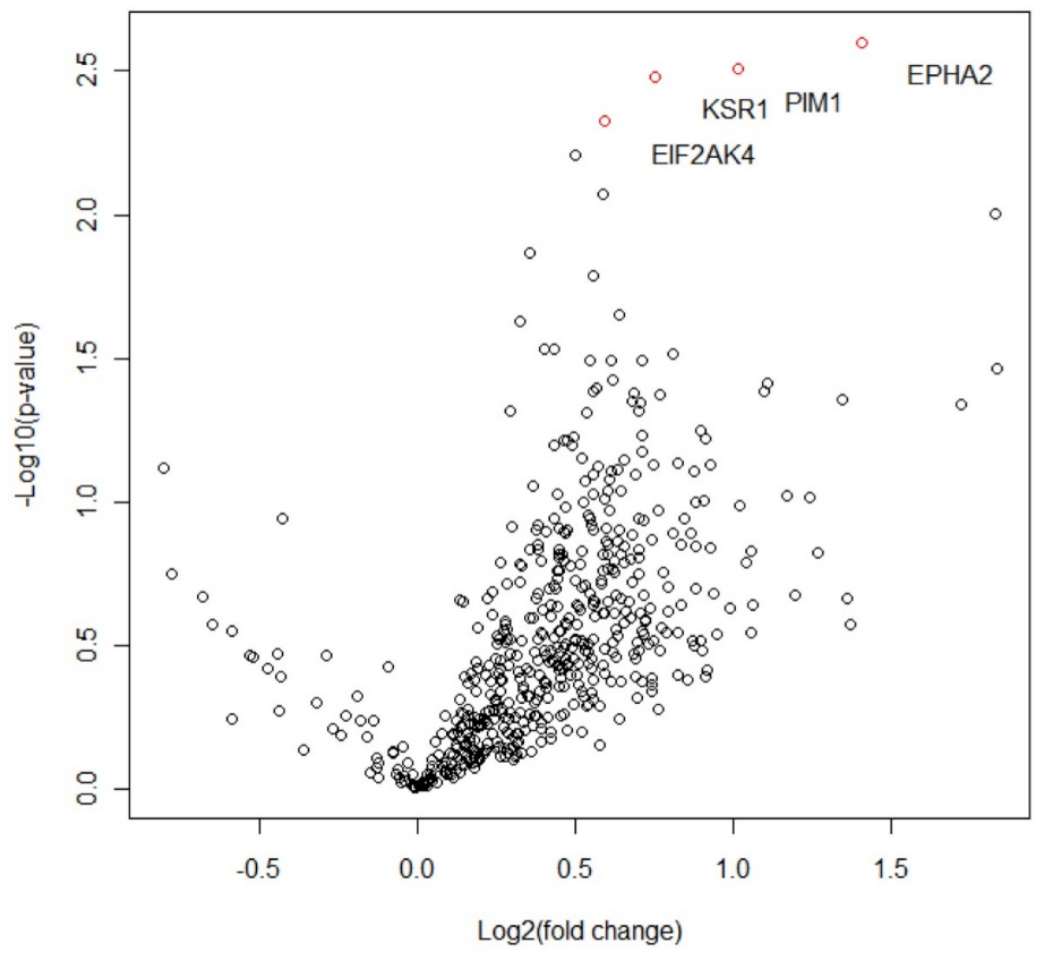

Figure 2. Among 519 kinase genes, 4 significant differentially expressed genes whose $P$-value $<0.005$ and their fold changes are shown in Volcano plot.

\section{Discussion}

The present study represents the first comprehensive high-throughput genomic analysis to identify biomarkers predictive of the response for RAD001 containing treatment in refractory GC patients. Based on our genomic analysis, there is significant overexpression of 4 genes such as EPHA2 ( $P=0.0025)$, PIM1 $(P=0.0031)$, KSR1 $(P=0.0033)$, and EIF2AK4 $(\mathrm{P}=0.0046)$ related to the response for capecitabine plus RAD001. These finding may help define subsets who would receive the most benefit from RAD001 treatment.

mTOR is a central regulatory kinase that increases the production of proteins that stimulate key cellular processes such as cell growth and proliferation, cell metabolism, and angiogenesis [16-18]. The mTOR pathway has been shown to be frequently dysregulated in a variety of human cancers, including gastric cancer [19]. Patient-derived gastric cancer samples have been shown to express phosphorylated mTOR indicative of mTOR activation, which has been positively correlated with tumor progression and poor survival in GC patients $[6,20-23]$. Our study showed there is significant overexpression of 4 genes such as EPHA2 ( $\mathrm{P}=0.0025)$, PIM1 $(\mathrm{P}=0.0031)$, KSR1 ( $\mathrm{P}=0.0033)$, and EIF2AK4 ( $\mathrm{P}$ $=0.0046)$ related to the response for capecitabine plus RAD001. Interestingly, the overexpression of these genes has been known to associate with the activation 
of mTOR-signaling (Table 5). Overexpression and mutation of EPHA2 were known to be associated with mTOR phosphorylation in lung cancer [24]. PIM1 was also reported in promoting mTOR activity leading to increased phosphorylation of two effector molecules, S6kinase 1 (S6K1) and 4E-binding protein 1 (4EBP1) by Kathleen et al, Zhang et al [25, 26]. KSR1, a scaffold protein for Raf, MEK and ERK plays a critical role in the optimal activation of ERK [27]. Furthermore, KSR1 is known to associate with mTOR. Thus, KSR1 regulates mTOR activation, both by controlling ERK activation and by bringing together members of ERK and the mTOR pathway [28-30]. Lastly, it has been known that ELF2AK helps S6K1 activated by mTOR to promote mRNA translation [31, 32]. Along these lines, four genes such as EPHA2, PIM1, KSR1, and EIF2AK4 have been known to be associated with the activation of mTOR signalling, directly or indirectly. Therefore, it is theoretically proper that patients with overexpression of these kinase genes benefit from RAD001-containing therapy in our study. As far as we know, this is first-study that reports the overexpression of EPHA2, PIM1, KSR1, and EIF2AK4 related to the activation of mTOR signalling in GC. These findings reconfirm that molecular targeted agent can be effective in only selected patients with the dependence on signal-pathway related to target-molecule.

Among expression nature of 519 kinase genes, overexpressed kinase genes related to the response for capecitabine plus RAD001 were sorted while changing the weight of p-value such as $0.05,0.01$, and 0,005 . In $\mathrm{P}<0.05$, overexpressed 32 kinase genes (EPHA2, PIM1, KSR1, EIF2AK4, SGK223, STK38, PNCK, CSNK2A2, TLK1, MAPK14, SGK3, ADCK2, MTOR, MAP4K3, TAF1L, NLK, PRKG1, EPHA7, SRPK2, MAPK11, MAPKAPK5, PRKY, STK35, PAK4, SGK494, PRKAA2, SIK2) and of 519 were selected and, in $\mathrm{P}<0.01,7$ kinase (EPHA2, PIM1, KSR1, EIF2AK4, SGK223, STK38 and PNCK) genes selected. However, sample size of this study was too small, and the stricter $p$-value $(\mathrm{P}<0.005)$ was needed for increasing the statistical significance and power. For the overexpression of mTOR, there was significant relation to successful response for RAD001 in $p$-value $<0.01$, but not in $\mathrm{P}<0.005$. Signal transduction

Table 5. Potential predictors and activation of mTOR signaling.

\begin{tabular}{llll}
\hline Authors & Kinase gene & Marker & Signaling \\
\hline Leonardi et al & EPHA2 & Overexpression, Mutation & mTOR activation \\
Kathleen et al, Zhang et al & PIM1 & Overexpression & mTOR activation \\
Shaw et al, Dougherty et al & KSR1 & Overexpression & mTOR activation \\
Howell et al & EIF & Activation & mTOR activation
\end{tabular}

through mTOR pathway is processed by both mTOR itself and various biologic network of upstream and downstream [33]. The intensity of signalling through mTOR pathway may be more affected by other kinase workings related to the pathway rather than overexpression of mTOR, itself. Furthermore, in addition to overexpressed kinase genes, other types of genetic alterations such as fusion and mutations might affect the activation or downregulation to signalling through mTOR pathway. Thus, due to the complexity of mechanisms underlying mTOR pathway activation, comprehensive genomic analysis of tumour is needed. Gene expression based pathway readout may be more appropriate than relying on a single indicator of pathway activity, as alterations in multiple signalling components could lead to pathway activation and result in similar downstream effects. Recent studies showed the potential for gene signature derived from platforms to be predictive of clinical drug response [34-36]. We plan to develop the signature based-platform to predict the response for RAD001 and validate it in our patients' derived gastric cells.

\section{Conclusions}

In conclusion, this study is first report that investigated to identify biomarkers predictive of the response for RAD001 containing treatment in refractory GC patients, by comprehensive high-throughput genomic analysis (encounter assay). Responders for RAD001 containing treatment had overexpression of kinase genes such as EPHA2, PIM1, KSR1, and EIF2AK4 related to activation of motor pathway. This comprehensive genomic analysis for motor pathway is useful to evaluate biomarkers for RAD001 as well as to find a new novel molecule potentially involved in mTOR signalling.

\section{Acknowledgements}

This work was supported by funding from the Korean Health Technology R\&D Project, Ministry of Health \& Welfare, Republic of Korea (HI14C2750, HI14C3418). Support was also provided by a grant from the Samsung Medical Center (SMX1161251).

\section{Authors' contributions}

All authors made substantial contributions to the conception and design of the study, and acquisition, analysis, and interpretation of the data. All authors were involved in drafting the manuscript (or revising it), and all read and approved the final manuscript. 


\section{Competing interests}

The authors declare that they have no competing interests.

\section{References}

1. Kamangar F, Dores GM, Anderson WF. Patterns of cancer incidence, mortality, and prevalence across five continents: defining priorities to reduce cancer disparities in different geographic regions of the world. J Clin Oncol. 2006; 24: 2137-2150.

2. Parkin DM, Bray F, Ferlay J, et al. Global cancer statistics, 2002. CA Cancer J Clin. 2005; 55: 74-108.

3. Van Cutsem E, Haller D, Ohtsu A. The role of chemotherapy in the current treatment of gastric cancer. Gastric Cancer. 2002; 5 Suppl 1:17-22.

4. Ji SH, Lim DH, Yi SY, et al. A retrospective analysis of second-line chemotherapy in patients with advanced gastric cancer. BMC Cancer. 2009; 9: 110.

5. Oki E, Baba H, Tokunaga E, et al. Akt phosphorylation associates with $\mathrm{LOH}$ of PTEN and leads to chemoresistance for gastric cancer. Int J Cancer. 2005; 117: 376-380.

6. Lang SA, Gaumann A, Koehl GE, et al. Mammalian target of rapamycin is activated in human gastric cancer and serves as a target for therapy in an experimental model. Int J Cancer. 2007; 120: 1803-1810.

7. Strimpakos AS, Karapanagiotou EM, Saif MW, et al. The role of mTOR in the management of solid tumors: an overview. Cancer Treat Rev. 2009; 35: 148-159.

8. Okamoto I, Doi T, Ohtsu A, et al. Phase I clinical and pharmacokinetic study of RAD001 (everolimus) administered daily to Japanese patients with advanced solid tumors. Jpn J Clin Oncol. 2010; 40: 17-23.

9. Doi T, Muro K, Boku N, et al. Multicenter phase II study of everolimus in patients with previously treated metastatic gastric cancer. J Clin Oncol. 2010; 28: 1904-1910.

10. Ohtsu A, Ajani JA, Bai YX, et al. Everolimus for previously treated advanced gastric cancer: results of the randomized, double-blind, phase III GRANITE-1 study. J Clin Oncol. 2013; 31: 3935-3943.

11. Yoon DH, Ryu MH, Park YS, et al. Phase II study of everolimus with biomarker exploration in patients with advanced gastric cancer refractory to chemotherapy including fluoropyrimidine and platinum. Br J Cancer. 2012; 106: 1039-1044.

12. Lee SJ, Lee I, Lee J, et al. Phase II trial of capecitabine and everolimus (RAD001) combination in refractory gastric cancer patients. Invest New Drugs. 2013; 31: 1580-1586.

13. Bu X, Le C, Jia F, et al. Synergistic effect of mTOR inhibitor rapamycin and fluorouracil in inducing apoptosis and cell senescence in hepatocarcinoma cells. Cancer Biol Ther. 2008; 7: 392-396.

14. Lee KH, Hur HS, Im SA, et al. RAD001 shows activity against gastric cancer cells and overcomes 5-FU resistance by downregulating thymidylate synthase. Cancer Lett. 2010; 299: 22-28.

15. Geiss GK, Bumgarner RE, Birditt B, et al. Direct multiplexed measurement of gene expression with color-coded probe pairs. Nat Biotechnol. 2008; 26: 317-325.

16. Fingar DC, Richardson CJ, Tee AR, et al. mTOR controls cell cycle progression through its cell growth effectors S6K1 and 4E-BP1/eukaryotic translation initiation factor 4E. Mol Cell Biol. 2004; 24: 200-216.

17. Edinger AL, Thompson CB. Akt maintains cell size and survival by increasing mTOR-dependent nutrient uptake. Mol Biol Cell. 2002; 13: 2276-2288.

18. Patel PH, Chadalavada RS, Chaganti RS, et al. Targeting von Hippel-Lindau pathway in renal cell carcinoma. Clin Cancer Res. 2006; 12: 7215-7220.

19. Bjornsti MA, Houghton PJ. The TOR pathway: a target for cancer therapy. Nat Rev Cancer. 2004; 4: 335-348.

20. Murayama T, Inokuchi M, Takagi Y, et al. Relation between outcomes and localisation of p-mTOR expression in gastric cancer. Br J Cancer. 2009; 100: 782-788.

21. Xiao L, Wang YC, Li WS, et al. The role of mTOR and phospho-p70S6K in pathogenesis and progression of gastric carcinomas: an immunohistochemical study on tissue microarray. J Exp Clin Cancer Res. 2009; 28: 152.

22. Yu G, Wang J, Chen $Y$, et al. Overexpression of phosphorylated mammalian target of rapamycin predicts lymph node metastasis and prognosis of chinese patients with gastric cancer. Clin Cancer Res. 2009; 15: 1821-1829.

23. An JY, Kim KM, Choi MG, et al. Prognostic role of p-mTOR expression in cancer tissues and metastatic lymph nodes in $\mathrm{pT} 2 \mathrm{~b}$ gastric cancer. Int J Cancer. 2010; 126: 2904-2913.

24. Faoro L, Singleton PA, Cervantes GM, et al. EphA2 mutation in lung squamous cell carcinoma promotes increased cell survival, cell invasion, focal adhesions, and mammalian target of rapamycin activation. J Biol Chem. 2010; 285: 18575-18585.

25. Zhang F, Beharry ZM, Harris TE, et al. PIM1 protein kinase regulates PRAS40 phosphorylation and mTOR activity in FDCP1 cells. Cancer Biol Ther. 2009; 8: 846-853.

26. Buckley K, Fiskus W, Bhalla K. Activation of mTOR: A new mechanism "PIMed". Cancer Biol Ther. 2009; 8: 854-855.
27. Shaw AS, Filbert EL. Scaffold proteins and immune-cell signalling. Nat Rev Immunol. 2009; 9: 47-56.

28. Giurisato E, Lin J, Harding A, et al. The mitogen-activated protein kinase scaffold KSR1 is required for recruitment of extracellular signal-regulated kinase to the immunological synapse. Mol Cell Biol. 2009; 29: 1554-1564.

29. Nguyen A, Burack WR, Stock JL, et al. Kinase suppressor of Ras (KSR) is a scaffold which facilitates mitogen-activated protein kinase activation in vivo. Mol Cell Biol. 2002; 22: 3035-3045.

30. Lin J, Harding A, Giurisato E, et al. KSR1 modulates the sensitivity of mitogen-activated protein kinase pathway activation in $\mathrm{T}$ cells without altering fundamental system outputs. Mol Cell Biol. 2009; 29: 2082-2091.

31. Gallinetti J, Harputlugil E, Mitchell JR. Amino acid sensing in dietary-restriction-mediated longevity: roles of signal-transducing kinases GCN2 and TOR. Biochem J. 2013; 449: 1-10.

32. Wang $X, \mathrm{Li} \mathrm{W}$, Williams M, et al. Regulation of elongation factor 2 kinase by p90(RSK1) and p70 S6 kinase. EMBO J. 2001; 20: 4370-4379.

33. Zoncu R, Efeyan A, Sabatini DM. mTOR: from growth signal integration to cancer, diabetes and ageing. Nat Rev Mol Cell Biol. 2011; 12: 21-35.

34. Loboda A, Nebozhyn M, Klinghoffer R, et al. A gene expression signature of RAS pathway dependence predicts response to PI3K and RAS pathway inhibitors and expands the population of RAS pathway activated tumors. BMC Med Genomics. 2010; 3: 26.

35. Dry JR, Pavey S, Pratilas CA, et al. Transcriptional pathway signatures predict MEK addiction and response to selumetinib (AZD6244). Cancer Res. 2010; 70: 2264-2273.

36. Bild AH, Yao G, Chang JT, et al. Oncogenic pathway signatures in human cancers as a guide to targeted therapies. Nature. 2006; 439: 353-357. 